\title{
Energy Efficiency Optimization for IR-UWB WBAN Based on the IEEE 802.15.6 Standard
}

\author{
Heikki Karvonen \\ heikki.karvonen@ee.oulu.fi
}

\author{
Jari linatti \\ jari.iinatti@ee.oulu.fi \\ Centre for Wireless Communications \\ University of Oulu \\ Oulu, Finland
}

\author{
Matti Hämäläinen \\ matti.hamalainen@ee.oulu.fi
}

\begin{abstract}
This paper describes a cross-layer energy efficiency optimization for the IEEE 802.15.6 standard based wireless body area networks. Studied physical layer is based on impulse radio ultra wideband signaling using non-coherent energy detection receiver and on-off keying modulation. Bose-Chaudhuri-Hocquenghem code is used for forward error correction and the analyzed medium access control layer is using slotted Aloha, which is a mandatory method for the contention based mode of the IEEE 802.15.6 standard. Proposed model can be used to explore the energy efficiency of uncoded and coded communication when using different payload lengths. Results clearly show the energy efficiency for different payload lengths as a function of signal-tonoise ratio in the additive white Gaussian noise channel.
\end{abstract}

\section{Categories and Subject Descriptors}

C.2.2 [Computer - Communication Networks]: Network Protocols; C.2.1 [Computer - Communication Networks]: Network Architecture and Design - Wireless Communication

\section{General Terms}

Performance, Design, Reliability, Theory.

\section{Keywords}

wireless body area network, impulse radio ultra wideband, crosslayer optimization.

\section{INTRODUCTION}

Wireless body area networks (WBAN) have various application possibilities, e.g., in the sports, healthcare, entertainment and military scenarios [1] - [3]. Therefore, recently an IEEE 802.15.6 standard [4] which is targeted for multiple WBAN applications

Permission to make digital or hard copies of all or part of this work for personal or classroom use is granted without fee provided that copies are not made or distributed for profit or commercial advantage and that copies bear this notice and the full citation on the first page. To copy otherwise, to republish, to post on servers or to redistribute to lists, requires prior specific permission and/or a fee.

UWBAN-2013 2013, September 30-October 02

Copyright (c) 2013 ICST 978-1-936968-89-3

DOI 10.4108/icst.bodynets.2013.253672 has been published. Standard defines three different physical (PHY) layer options and medium access control (MAC) modes, which characteristics are suitable for varying application scenarios. On-off keying (OOK) based signaling, which enables non-coherent detection, has been chosen as the mandatory option for impulse radio ultra wideband (IR-UWB) PHY case [4]. Noncoherent detection enables simple and low power IR-UWB based WBAN transceiver structures. Also the MAC protocol has significant effect to the energy efficiency of communication [5]. UWB communication sets special challenges for the MAC design because signals are noise-like and traditional carrier sensing methods cannot be applied. However, MAC protocol should enable that the number of retransmissions can be minimized and the channel access can be done efficiently. In the IEEE 802.15.6 standard, the MAC protocol design challenges have been solved by defining different access phases (contention free and contention based) for superframe and different methods for channel completion [4]. In the IR-UWB case, slotted Aloha is used for contention based channel access [4]. Therefore the energy efficiency of IR-UWB PHY and slotted Aloha MAC combination will be explored here.

There are few related works addressing networks based on the IEEE 802.15.6 standard. Model for throughput, delay and bandwidth efficiency analysis as a function of payload length has been introduced in [6]. An energy analysis for scheduled access mode has been introduced in [7]. However, UWB PHY is not considered and ideal channel conditions have been assumed in [6] and [7]. IEEE 802.15.6 standard based WBAN using narrowband PHY has been analyzed in [8] by assuming saturation condition. In [9] and [10] narrowband PHY study has been done for nonsaturation condition. In [11], simulation results for bit-interleaved UWB on-off waveform coded modulation are shown and simple receiver architecture is proposed. In [12], authors have explored the energy optimal Reed-Solomon code rate for IR-UWB communication using binary burst position modulation (BPM) and non-coherent receiver. In [13] authors have analyzed the energy efficiency of the IEEE 802.15.6 standard based IR-UWB communication using energy detection (ED) receiver with different Bose-Chaudhuri-Hocquenghem $(\mathrm{BCH})$ code rates and payload lengths. This work takes advantage of the model developed in [13] and provides new results for varying payload lengths when using the code rate defined in the standard and for the uncoded case. In [13], a joint code rate and payload length optimization is performed. Here the model introduced in [13] is 
modified to focus more on the payload length optimization. The goal is that the results given here can be more easily applied to practical implementation without modification to the standard. While the joint optimization done in [13] is more useful if also the code rate can be changed to be different than in the standard.

The remainder of the paper is organized as follows. Section II introduces the system model and Section III provides description about the optimization model. Section IV introduces the results and the conclusions are done in Section VI.

\section{SYSTEM MODEL}

In this paper a star topology network, which is composed of $N$ nodes and one hub (sink), is used. Hub receives data from the nodes in a one hop communication fashion which is typically used in WBANs. However, standard [4] defines also an option for a two-hop star topology in which the nodes can also act as a relay. In this work the two-hop communication will not be particularly analyzed, but the introduced energy consumption model does not exclude the usage of communication between a node and relaynode in the WBAN.

Three PHY layer options have been defined for WBANs in [4]: a narrowband, UWB and human body communications (HBC) PHY. In this work, the IR-UWB with OOK modulation and ED receiver will be studied because it enables low cost and low power implementations. The non-coherent ED receiver operation is such that after the receiver antenna, the signal goes through a band-pass zonal filter (BPZF), which eliminates the out-of-band noise. The ED part of the receiver applies the square law operation to the filtered signal and the integrator is used to capture the signal energy which includes also noise. The integration time affects the amount of captured signal energy and noise. If the integration time is longer than symbol duration at the receiver input, then superfluous noise will be collected. On the other hand, too short integration time will lead to a loss of available signal energy. The symbol decision is made by comparing the amount of received energy in both symbol intervals in order to determine whether the transmitted bit was one or zero.

Three different channel access modes have been defined in [4]: a beacon mode with beacon periods (superframes); non-beacon mode with superframes; non-beacon mode without superframes. Here the focus is on the beacon mode with superframes. Hub shall establish a time base dividing the time axis into beacon periods, and it can set the lengths for different access phases (exclusive, random, managed and contention) depending on the predominant communication requirements in the network [4]. A second beacon needs to be send by the hub during superframe if the contention access phase (CAP) length is non-zero. The studied S-Aloha protocol is used in the contention based access phases. In slotted Aloha case the random access period is divided into slots in which the nodes shall transmit their frames. Node transmission probability in a given slot depends on the channel contention probability. The hub shall transmit a beacon message in the beginning of the superframe period so that the nodes know when they should compete for channel access. Standard defines different slotted Aloha channel access contention probabilities $C P_{\min } \leq C P \leq C P_{\max }$ for different access phases and they depend on the user priority [4]. During the channel competition nodes will adapt their $\mathrm{CP}$, according to the definitions of [4], depending on whether the transmission was successful or not.

\section{OPTIMIZATION MODEL}

The IR-UWB physical layer symbol structure shall be formed using a single pulse, or burst of pulses, waveform of duration $T_{w}=$ $N_{c p b} T_{p}$, which is defined as

$w(t)=\sum_{i=0}^{N_{c p b}-1} p\left(t-i T_{p}\right)$,

where $N_{c p b} \geq 1$ is the number of pulses per burst and $T_{p}$ is the pulse duration [4]. Standard [4] defines that the OOK signaling will be used in conjunction with $M$-ary waveform coding. The waveform coding maps $K$ information bits from an alphabet of size $M=2^{K}$ onto coded-pulse sequences of length $2 K$ from a code set alphabet of the same size $2^{K}$. In the mandatory mode of IRUWB PHY, $K=1$ and optional mode shall use $K=4$. I.e., the symbol time coincides with the binary pulse position modulation (BPPM) symbol time in the mandatory mode.

The OOK modulated signal will include pulse waveform when the input bit is one, i.e., for the $m^{\text {th }}$ symbol it can be expressed as

$x^{m}(t)=\sum_{n=0}^{2 K-1} d_{n}^{m} w_{2 K m+n}\left(t-n\left(T_{s y m} / 2\right)-m K T_{s y m}-h^{(2 K m+n)} T_{w}\right)$,

where $m \geq 0, d_{n}^{m}$ is the $n$ :th code word component of the $m$ :th symbol, $T_{s y m}$ is the symbol duration, $h^{(2 K m+n)}$ is the time hopping sequence and $w_{2 K m+n}(t)$ is defined in (1). The uncoded data rate for OOK modulation can be calculated as $R=1 / T_{\text {sym }}$. The scrambling and time hopping can be ignored in the bit error probability derivation of interference free additive white Gaussian noise (AWGN) channel used hereafter. The transmitted signal for the $m^{\text {th }}$ symbol is then defined as

$$
\begin{aligned}
& s^{m}(t)=\sum_{n=0}^{2 K-1} d_{n}^{m} \times \\
& \sum_{i=0}^{N_{c p b}-1} \sqrt{\frac{E_{s}}{N_{c p b} T_{p}}} p\left(t-n\left(T_{s y m} / 2\right)-m K T_{s y m}-i T_{p}\right),
\end{aligned}
$$

where $E_{s}$ is the energy per symbol, i.e., the energy per bit in binary modulation case.

Theoretical bit error probability (BEP) in the AWGN case for OOK signaling is calculated here by using the approach introduced for ED receiver with pulse position modulation [14]. It can be used here because in the OOK modulation combined with 2-ary waveform coding case, the symbol decision can be done by comparing the received signal energies of two different symbol intervals. The signal-to-noise ratio (SNR) at the decision variable of ED receiver can be then calculated as [14]

$$
S N R_{D V}=\frac{2 \frac{E_{b}^{i}}{N_{0}}}{4+N_{c p b} 2 \tau W \frac{N_{0}}{E_{b}^{i}}},
$$

where $\tau$ is the integration time per pulse, $W \approx 1 / T_{p}$ is the signal bandwidth, $E_{b}^{i}$ is the integrated energy per bit and $N_{0}$ is the onesided noise power spectral density per $\mathrm{Hz}$.

\subsection{PHY layer success probability}

In this paper, the performance of uncoded and coded communication will be analyzed. In the uncoded communication case the bit error probability, $P_{b}$, can be calculated by using (4) 
and the $\mathrm{Q}(x)$ function when assuming Gaussian approximation [14] for AWGN channel. In the multi-user interference case the Gaussian approximation is not valid because it leads to too optimistic results [15], [16].

The IEEE 802.15.6 standard defines that a BCH code shall be used in the default mode with following parameters [4]: total number of bits in a code word $n=63$; number of information bits $k=51$; error correction capability $t=2$. In order to have a fair comparison for uncoded and coded cases, each transmitted packet must contain the same amount of bit energy. Therefore, the energy per bit in coded case is $E_{c}=r E_{b}$, where $E_{b}$ is the energy per bit in the uncoded case and $r$ is the $\mathrm{BCH}$ code rate [17], [18]. The bit error probability before error correction at the decoder can be calculated as [18]

$$
P_{b d}=\mathrm{Q}\left(\sqrt{S N R_{D V}^{c}}\right) \text {, }
$$

where $S N R_{D V}^{c}$ is the signal-to-noise ratio at the decision variable when using $\mathrm{BCH}$ coding. Decoder can correct $t$ bit errors, therefore the code word error probability can be calculated as [17] [18]

$$
P_{c w} \approx \sum_{h=t+1}^{n}\left(\begin{array}{l}
n \\
h
\end{array}\right) P_{b d}{ }^{h}\left(1-P_{b d}\right)^{n-h} .
$$

The bit error probability for coded case can be approximated as [18]

$$
P_{c} \approx \frac{1}{n} P_{c w}=\frac{1}{n} \sum_{h=t+1}^{n}\left(\begin{array}{l}
n \\
h
\end{array}\right) P_{b d}{ }^{h}\left(1-P_{b d}\right)^{n-h} .
$$

The packet error probability for the packet length of $l$ bits can be calculated from the bit error probability, $P_{b}$, as $P E P=1-\left(1-P_{b}\right)^{l}$,

where $l$ is the length of the packet in bits in the uncoded case. When using the BCH code the packet length is $l_{c}=l / r$ and BEP for coded case $\left(P_{c}\right)$ can be derived using (7).

The physical layer success probability is defined here as

$P_{\text {succ }}^{P H Y}=1-P E P$.

In the coded case, it must be taken also into account that for certain packet lengths there is a need for bit filling in order to align with the number of information bits $(k)$ required in the last code word encoding as defined in standard [4].

\subsection{MAC layer success probability}

In the cross-layer analysis carried out in this work the channel access success probability will also be taken into account. For SAloha case, the MAC layer success probability can be calculated as

$P_{\text {succ }}^{M A C}=N p_{i}\left(1-p_{i}\right)^{N-1}$,

where $N$ is the number of nodes competing for the channel and $p$ is the probability, for a node $i$, to transmit in a slot. The maximum success probability in the S-Aloha case will be achieved when the offered traffic load $G=1$, which corresponds to case $p_{i}=1 / N$. [4] defines different probability values $p_{i}$, which depend on the user priority value and back-off state. Here it has been assumed that $p_{i}$ $=1 / N$, i.e., the channel competition probability is optimal for slotted Aloha. However, results for different traffic loads can be calculated as well by using the introduced model. The S-Aloha slot structure consists of data / management frame transmission, short interframe spacing and acknowledgement (ACK) frame transmission times [4]. Here it has been assumed that the slot duration is such that the data frame and ACK can be transmitted during the slot. Standard [4] enables this because the hub can set the superframe and slot lengths according to network requirements.

\subsection{Energy consumption model}

This work takes advantage of an energy consumption model introduced in [13]. From the energy consumption point of view, the number of required retransmissions and the packet duration are the key factors. The number of retransmissions depends on the PHY layer success probability and on the MAC layer success probabilities introduced in previous sections. Therefore, the number of expected transmissions required for successful packet reception can be calculated as

$$
\mathrm{E}\left\{N_{t x}\right\}=\frac{1}{P_{\text {succ }}^{P H Y} P_{\text {succ }}^{M A C}} .
$$

The energy consumption depends on the time used for frame transmission and reception. The transmitted UWB frame duration can be calculated as

$T_{\text {frame }}=T_{\text {SHR }}+T_{\text {PHR }}+T_{P S D U}$,

where $T_{S H R}$ is the duration of synchronization header (SHR), $T_{P H R}$ is the duration of physical layer header (PHR) and $T_{P S D U}$ is the duration of physical-layer service data unit (PSDU) which length depends on the error correction code rate [4]. The length of the synchronization and physical layer header does not depend on the coding.

Here has been assumed an immediate acknowledgement (I-ACK) mode in which the hub will transmit ACK right after the successful packet reception [4]. Therefore, after the data packet transmission, the transmitter will wait for the ACK message for duration of

$T_{\text {ACKwait }}=p S I F S+T_{S H R}+T_{P H R}+T_{A C K}$,

where $p S I F S$ is the short interframe spacing time and $T_{A C K}$ is the duration of the ACK packet. In this work it is assumed that the ACK message will always be received correctly, i.e., only the erroneous data packets will be retransmitted.

The energy consumption required for successful packet transmission and reception is a function of SNR $(\gamma)$ and payload length $(\lambda)$. Tx-Rx link energy consumption is calculated here as

$$
\begin{aligned}
& \mathcal{E}(\gamma, \lambda)=\left(\mathrm{E}\left\{N_{t x}\right\}-1\right)\left(T_{\text {frame }}\left(P_{t x, R F}+P_{t x, \text { circ }}\right)\right. \\
& \left.+E_{\text {enc }}+T_{A C \text { Kwait }} P_{r x}\right) \\
& +T_{\text {frame }}\left(P_{t x, R F}+P_{t x, \text { circ }}\right)+E_{e n c}+T_{A C K} P_{r x} \\
& +\mathrm{E}\left\{N_{t x}\right\}\left(T_{\text {frame }} P_{r x}+E_{d e c}\right)+T_{A C K}\left(P_{t x, R F}+P_{t x, C I R C}\right),
\end{aligned}
$$

where $P_{t x, R F}$ is transmitter RF power consumption, $P_{t x, c i r c}$ is transmitter circuitry power consumption, $P_{r x}$ is receiver power consumption, $E_{e n c}$ and $E_{d e c}$ are the encoding and decoding energies, respectively. Assume that Berklekamp Massey and Chien search algorithms are used at the decoder. Then for a $t$ error correcting $\mathrm{BCH}$ code of length $n$, the encoding and decoding energy consumption derivations are given in [13] and in [19]. In the uncoded case $E_{\text {enc }}=E_{d e c}=0$. 


\subsection{Energy efficiency metric}

The energy efficiency metric defined in [13] is the number of successfully received information bits per Joule as

$\eta=\frac{\lambda}{\varepsilon}$

where $\lambda$ is the number of information bits (payload length) and $\varepsilon$ is the energy consumption used for communicating the bits successfully from transmitter to receiver. In this paper the same definition for energy efficiency comparison will be used. In order to enable simple performance comparison, the energy efficiency will be normalized so that maximum value is one. The normalization is done with respect to maximum achievable energy efficiency in the Tx-Rx link as

$\eta_{\text {norm }}(\gamma, \lambda)=\frac{\eta(\gamma, \lambda)}{\max (\eta)}$

It is obvious, that if SNR is high enough to guarantee error free reception at the given link, then minimum energy consumption can be achieved with uncoded transmission. The rationale is that uncoded transmission includes minimum amount of overhead to communicate the $\lambda$ information bits. Let's define that the error free transmission and reception in the uncoded case can be achieved when SNR is $\gamma_{0}$ and payload is $\lambda_{\max }$. The normalized energy efficiency for particular payload length and SNR, can be then calculated as

$\eta_{o v, \text { norm }}(\gamma, \lambda)=\frac{\eta(\gamma, \lambda)}{\eta\left(\gamma_{0}, \lambda_{\max }\right)}=\frac{\varepsilon\left(\gamma_{0}, \lambda_{\max }\right)}{\varepsilon(\gamma, \lambda)}$.

I.e., eq. (17) gives normalization of energy efficiency in case of $\gamma$ with payload length $\lambda$ in comparison to efficiency that can be achieved with maximum payload and SNR which guarantees the error free reception for uncoded case.

\section{RESULTS}

This section shows the results calculated by implementing the introduced analytical model in Matlab. Parameters used for the calculations are shown in the Table I, and they are drawn from the definitions of the standard [4] and characteristics of the state of the art transceivers.

Figure 1 show the normalized energy efficiency as a function of $E_{\mathrm{b}} / N_{0}$ and payload length $\lambda$. The maximum energy efficiency can be achieved when the $E_{\mathrm{b}} / N_{0}$ is high enough to guarantee error free transmission and the maximum payload length is in use to minimize the transmission overhead. The maximum energy efficiency value (one) is then achieved when using the uncoded transmission in the highest $E_{\mathrm{b}} / N_{0}$ and longest payload case, as can be seen from Figure 1. Results also shows the trend how coding improves the energy efficiency when $E_{\mathrm{b}} / N_{0}$ decreases and payload length increases. For short payload the coding improves the energy efficiency less than for long payload. Following results figures will illustrate that behavior in more details for selected payload lengths.
Table 1. Parameters for performance evaluation

\begin{tabular}{|l|l|l|}
\hline Parameter & Description & Value \\
\hline$W$ & bandwidth & $499.2 \mathrm{MHz}$ \\
\hline$f_{c}$ & central frequency & $3993.6 \mathrm{MHz}$ \\
\hline$N_{c p b}$ & number pulses per bit & 16 \\
\hline$T_{p}$ & pulse duration & $2 \mathrm{~ns}$ \\
\hline$\tau$ & integration time & $2 \mathrm{~ns}$ \\
\hline$R$ & uncoded data rate & $0.975 \mathrm{Mbps}$ \\
\hline$I$ & implementation losses & $5 \mathrm{~dB}$ \\
\hline$N F$ & receiver noise figure & $10 \mathrm{~dB}$ \\
\hline$P_{t x, R F}$ & Tx RF power consumpt. & $37 \mu \mathrm{W}$ \\
\hline$P_{t x, \text { circ }}$ & Tx circuitry power consumpt. & $2 \mathrm{~mW}$ \\
\hline$P_{r x}$ & Rx power consumption & $20 \mathrm{~mW}$ \\
\hline$P_{s d}$ & maximum signal power & $-41 \mathrm{dBm} / \mathrm{MHz}$ \\
\hline$N_{O}$ & thermal noise power & $-174 \mathrm{dBm} / \mathrm{Hz}$ \\
\hline$N_{M A C h e a d e r}$ & MAC header length & 7 octets \\
\hline$N_{F C S}$ & frame check sequence length & 2 octets \\
\hline$T_{S H R}$ & duration of synch. header & $40.32 \mu \mathrm{s}$ \\
\hline$T_{P H R}$ & duration of PHY layer header & $82.052 \mu \mathrm{s}$ \\
\hline$p S I F S$ & short interframe spacing & $75 \mu \mathrm{s}$ \\
\hline$N$ & number of nodes in WBAN & 10 \\
\hline$\lambda$ & payload length & $5-255 \mathrm{oct}$. \\
\hline & & \\
\hline
\end{tabular}

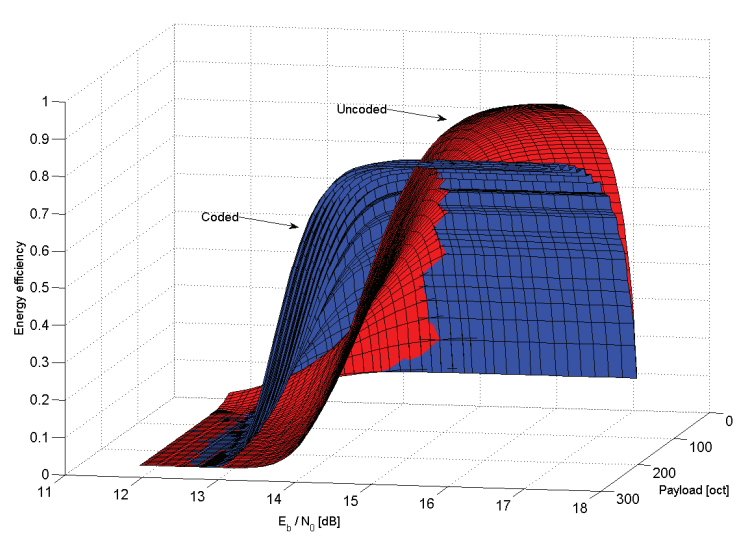

Figure 1. Normalized energy efficiency as a function of payload length and $E_{\mathrm{b}} / N_{0}$ in $\mathrm{dB}$. 


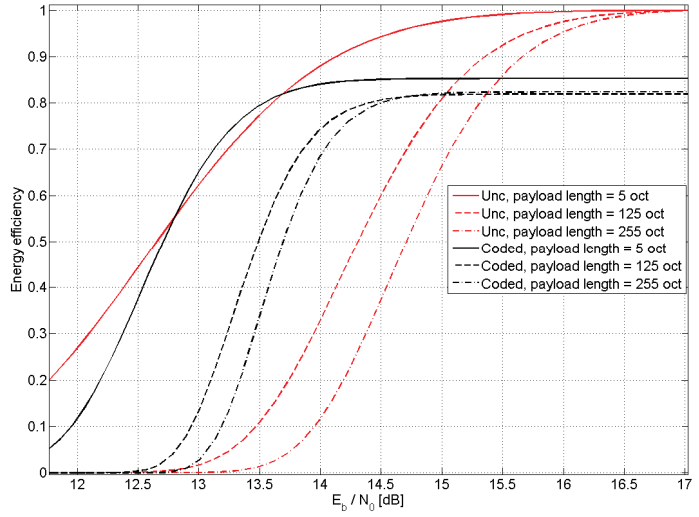

Figure 2. Energy efficiency for uncoded and coded cases with different payload lengths as a function of $E_{\mathrm{b}} / N_{0}$ in $\mathrm{dB}$.

Figure 2 shows energy efficiency results for selected payload values as a function of $E_{\mathrm{b}} / N_{0}$ in $\mathrm{dB}$. Results clearly illustrate that when $E_{\mathrm{b}} / N_{0}$ is high, the uncoded case will lead to highest energy efficiency. However, when the $E_{\mathrm{b}} / N_{0}$ decreases, coding starts to be useful. From the results it can be observed how the energy efficiency superiority between uncoded and coded case depends on the payload length. If payload length $(\lambda)$ is 125 octets, the uncoded case will lead to highest energy efficiency when $E_{\mathrm{b}} / N_{0}$ is above $15.2 \mathrm{~dB}$. However, if $E_{\mathrm{b}} / N_{0}$ is only $13.5 \mathrm{~dB}$, the coded case would lead to five times higher energy efficiency for the same payload length. It can be observed that especially for longer payload lengths, the coded case will improve the energy efficiency remarkably with $E_{\mathrm{b}} / N_{0}$ values lower than $14.5 \mathrm{~dB}$. On the other hand if $E_{\mathrm{b}} / N_{0}$ is high, uncoded case is more energy efficient also in long payload cases.

Figure 3 shows in percentage how much coding improves the energy efficiency in comparison to uncoded case for different payload lengths. It can be observed that for long payload cases the energy efficiency improvement is drastic when $E_{\mathrm{b}} / N_{0}$ is between 12.3 and $14.5 \mathrm{~dB}$. Results show also that when the payload is very short, the energy efficiency improvement of coding diminishes.

As can be seen from the results of Figure 1 - Figure 3, the energy efficiency depends highly on the payload length. Therefore, Figure 4 shows the average energy efficiency for payload lengths between 5 and 255 octets. This result show that if the payload lengths vary randomly, according to uniform distribution between 5 and 255 octects, the coding will improve the energy efficiency if $E_{\mathrm{b}} / N_{0}$ is below $15.2 \mathrm{~dB}$. It can be observed that even the short payload length do not need error correction, the averaged saving is remarkable because the energy efficiency is improved so drastically for the long payload cases. Results show also that if the $E_{\mathrm{b}} / N_{0}$ is too low (approximately below $12.3 \mathrm{~dB}$ ), error correction coding starts to be harmful because too many errors occur and they cannot be corrected. However, so low $E_{\mathrm{b}} / N_{0}$ would lead to very inefficient communication also in the uncoded case and that is not the feasible operation point for the transceivers.

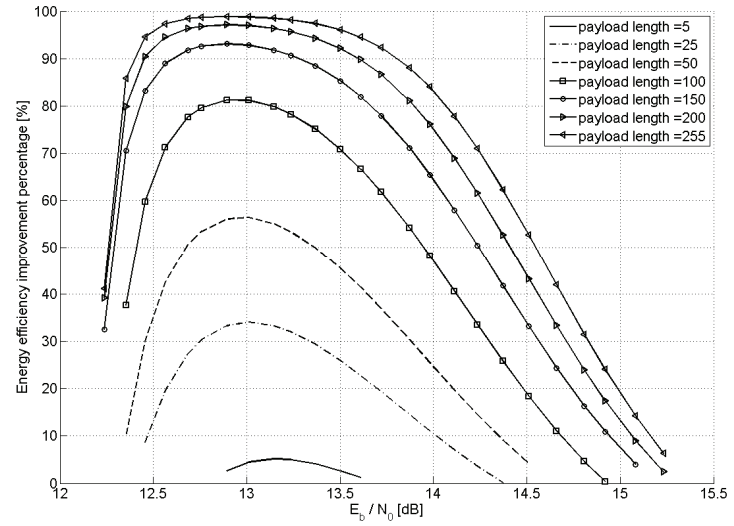

Figure 3. Energy efficiency improvement percentage of coded versus uncoded case as a function of $E_{\mathrm{b}} / N_{0}$ in $\mathrm{dB}$.

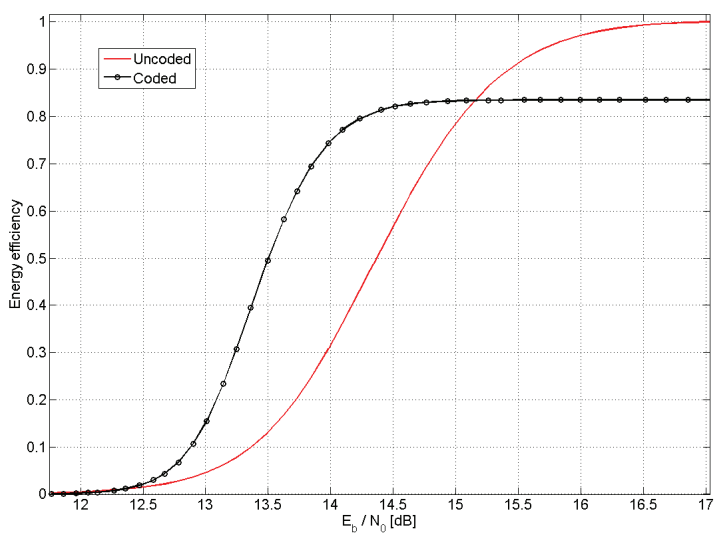

Figure 4. Energy efficiency averaged over payload length of 5 and 255 octets as a function of $E_{\mathrm{b}} / N_{0}$ in dB.

\section{CONCLUSIONS}

Energy efficiency optimization model for the IEEE 802.15.6 standard based IR-UWB WBAN physical and MAC layers was introduced in this paper. Optimization is done in a cross-layer fashion to explore energy efficiency when using different payload lengths in uncoded and coded communication. Non-coherent ED receiver is used for OOK modulated signal detection. Results clearly showed the energy efficiency as a function of $E_{\mathrm{b}} / N_{0}$ in $\mathrm{dB}$ for varying payload lengths. It was found that energy efficiency depends highly on the payload length and error correction coding. It can be concluded that the error correction improves energy efficiency when $E_{\mathrm{b}} / N_{0}<15.2 \mathrm{~dB}$ in the analyzed AWGN channel case. Moreover, it can be concluded that for very short messages the error correction should not be used from the energy efficiency point of view. In the future research more detailed channel models and measurements could be used to explore the performance in real environments. Introduced optimization approach and its results can be used, for example, to build radio protocol stacks reconfiguration and adaptation algorithms. 


\section{ACKNOWLEDGMENTS}

The work has been funded by Academy of Finland by the project Dependable Wireless Healthcare Networks for Reliable Body Area Network-based Healthcare (DWHN).

\section{REFERENCES}

[1] Huasong, C., Leung, V., Chow, C., and Chan, H. 2009. Enabling technologies for wireless body area networks: A survey and outlook. IEEE Communications Magazine, 47, 12 (Dec. 2009), 84 - 93.

[2] Latre, B., Braem, B., Moerman, I., Blondia, C., and Demeester, P. 2011. A survey on wireless body area networks. Springer Wireless Networks Journal, 17, 1 (Jan. 2011), 1-18.

[3] Ullah, S., et.al. 2012. A comprehensive survey of wireless body area networks. Springer Science + Business Media, Journal of Medical Systems, 36, 3 (Jun. 2012), 1065-1094.

[4] IEEE STD. 802.15.6. 2012. IEEE Standard for Local and metropolitan area networks - Part 15.6: Wireless Body Area Networks. The Institute of Electrical and Electronics Engineers, Inc.

[5] Boulis, A., Smith, D., Miniutti, D., Libman, L., and Tselishchev, Y. 2012. Challenges in body area networks for healthcare: the MAC. IEEE Communications Magazine, 50, 5 (May 2012), 100-106.

[6] Ullah S., and Kwak, K. S. 2011. Throughput and delay limits of IEEE 802.15.6. IEEE Wireless Communications \& Networking Conference (WCNC, Mar. 2011).

[7] Tachtatzis, C., Di Franco, F., Tracey, D.C., Timmons, N.F., and Morrison, J. 2010. An energy analysis of IEEE 802.15.6 scheduled access modes. IEEE Global Communications Conference (GLOBECOM, Dec. 2010).

[8] Rashwand, S., Misic, J., and Khazaei, H. 2011. IEEE 802.15.6 under saturation: some problems to be expected. Journal of Communications and Networks, 13, 2 (Apr.2011), 142-148.

[9] Rashwand, S., and Misic, J. 2011. Performance evaluation of IEEE 802.15.6 under non-saturation condition. IEEE Global Communications Conference (GLOBECOM, Dec. 2011).
[10] Jung, B. H., Akbar R. U., and Sung, D. K. 2012. Throughput, energy consumption and energy efficiency of IEEE 802.15.6 body area network (BAN) MAC protocol. International Symposium on Personal, Indoor and Mobile Radio Communications (PIMRC, Sep. 2012).

[11] Hernandez, M. and Kohno, R. 2011. UWB on-off waveform coded modulation for body area networks. IEEE International Conference on Ultra-Wideband (ICUWB, Sep. 2011).

[12] Karvonen, H., and Goratti, L. 2010. Optimal code rate for wireless sensor networks using IR-UWB and non-coherent detection. IEEE International Conference on Mobile Ad-hoc and Sensor Systems (MASS, Nov. 2010).

[13] Karvonen, H., Iinatti, J., and Hämäläinen, M. 2013. Crosslayer optimization model for IR-UWB based WBAN," Springer Telecommunication Systems Journal Special Issue on Research Advances in Energy-Efficient MAC Protocols for WBANs (Accepted for publication, March 2013).

[14] Rabbachin, A. 2008. Low Complexity UWB Receivers with Ranging Capabilities. Doctoral Thesis. University of Oulu. http://herkules.oulu.fi/isbn9789514288005/isbn9789514288 005.pdf.

[15] Durisi, G., and Romano, G. 2002. On the validity of Gaussian approximation to characterize the multiuser capacity of UWB TH-PPM. IEEE Conference on Ultra Wideband Systems and Technologies (UWBST, May 2002).

[16] Fiorina, J., Domenicali, D. 2009. The non validity of the gaussian approximation for multi-user interference in ultra wide band impulse radio: from an inconvenience to an advantage - transactions papers. IEEE Transactions on Wireless Communications, 8, 11 (Nov. 2009), 5483-5489.

[17] Lin, S., and Costello, D. J. Jr. 1983. Error control coding: Fundamentals and Applications. Prentice-Hall, Inc. Englewood Cliffs, New Jersey 07632.

[18] Proakis, J. G. 2001. Digital Communications 4th Edition. McGraw-Hill, Avenue of the Americas, New York 10020.

[19] Goel, M. and Shanbhag, N. R. 1999. Low power channel coding via dynamic reconfiguration. IEEE International Conference on Acoustic, Speech, and Signal Processing (ICASSP, Mar. 1999). 